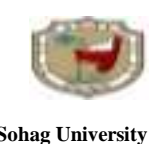

\title{
Impact of Radiofrequency ablation in the management of symptomatic uterine myoma
}

\section{Elsemary M.A ${ }^{1}$, Sabri M.M ${ }^{1}$, Abdelmonem A ${ }^{1}$. M, Ibrahim $\mathbf{M}^{2}$., Hisham A.Alghany Algahlan'.}

1-obstetrics and gynecology department, faculty of medicine, Sohag University, Egypt.

2-Radiology department, Faculty of Medicine, Sohag University, Egypt.

\section{Abstract:}

\section{OBJECTIVES:}

To evaluate the feasibility and safety of Ultrasound-guided radiofrequency ablation in the treatment of women with symptomatic uterine fibroids.

DESIGN: Prospective interventional clinical trial study.

METHODS: Forty women with symptomatic uterine fibroids underwent Ultrasoundguided radiofrequency ablation. Primary Outcome measures are changes in fibroidrelated symptoms and tumor size after the procedure measured at baseline, 3 and 6 months after the intervention. Secondary Outcome measures are a recurrence of fibroid symptoms following the procedure ( Time Frame: Baseline to 6 months ) and operative complications ( Time Frame: Baseline to 6 weeks ).

RESULTS:

The 40 patients, fulfilled the follow-up evaluations post-ablation. The mean uterine fibroid volume reduction rate was $40 \%$ at 3 months and $60 \%$ at 6 months post-ablation. symptoms, especially bleeding were statistically significantly declined. No severe complications such as penetration or burn injuries of the nearby organs were detected.

CONCLUSION:

Ultrasound-guided radiofrequency ablation is effective in reducing fibroid symptoms and size. Ultrasound-guided radiofrequency ablation is a promising new treatment option for Leiomyomas and should be further evaluated.

\section{Introduction:}

The most common benign tumors of the female genital system are uterine myomas (1).

They are benign tumors arising from the smooth muscle cells of the myometrium and composed of plentiful amounts of an extracellular matrix having fibronectin, collagen, and proteoglycan (2). The pathophysiology is indistinct, enigmatic and multiple factors (3).
They represent $20-40 \%$ of women during their reproductive ages. Because of racial differences (4).

About $50 \%$ of women are asymptomatic, however, leiomyomas can lead to major morbidity and worsening life quality (5). Symptomatic myomas can lead to abnormal uterine hemorrhage, pressure symptoms, and impacts on fertility, pregnancy, and labor.

Diagnosis is made by physical examinations, clinical doubt of myoma 
is recognized by c imaging techniques. Abdominal and Vaginal ultrasounds are frequently the technique of the first choice because of obtainability and little expenses.

MRI is the procedure of choice when accurate charting of the myoma is required before surgical scheduling.CT has inadequate sensitivity in detecting myomas (6).

Up till now, medical and surgical management are obtainable and their selection determined by the size, number, and site of myomas, age of the patient and favorites, and anticipations of pregnancy.

Though hysterectomy is an efficient method, slightly invasive treatment of uterine fibroids has acquired attractiveness as an alternative to conventional surgical excision. Minimally invasive methods include laparoscopic myomectomy, embolization of uterine artery(UAE), and thermal ablation techniques with the use of temperature to destroy tissue, including high-intensity focused ultrasound (HIFU), thermal ablation myolysis, cryomyolysis, laparoscopic-guided laser ablation, microwave and radiofrequency thermal ablation

One of the methods of hyperthermic ablation is the radiofrequency ablation (RFA) i.e., tissue destruction by using increased temperature. Heat might be utilized by direct thermal transmission or by electromagnetic or ultrasound (radiofrequency, microwave, laser) energy.

A form of applying energy for RFA is an alternating current in the radiofrequency range (between $3 \mathrm{kHz}$ and $300 \mathrm{GHz}$ ). Frequencies between 450 to $500 \mathrm{kHz}$ are used for medical procedures. Stimulation of nerves does not occur at frequencies below $10 \mathrm{kHz}$ during radiofrequency ablation (7).

In general, RFA is, effective, reliable and predictable, given a proper target tissue.

Generally, the radiofrequency device comprises a generator, a needle probe, probe return pads, and cables joining these structures.

A low voltage, high-frequency alternating current is produced by the generator that is transferred through a probe with an isolated shaft. After the settlement of the needle into the aimed tissue, the spread of the current via the tissue occurs with a specific point of entrance, Then the current spreads to the electrode return pads and then to the generator, closing the circuit. Production of heat is produced by Ionic $(\mathrm{Na}, \mathrm{Cl}, \mathrm{K})$ movements (resistive heating) inside the cells next to the uncovered part of the probe in reply to the very fast shifts in polarity integral with the alternating current, which then diffuses by simple thermal transmission, creating an ablation via coagulation necrosis. The volume, shape, and size of ablation are characterized by the temperature attained, the time extent at that temperature, and the form of the probe. Tissue transmission and the existence or absence of fluid collections or increased vascularity which acts as "heatsinks" are further factors that affect ablation.

At $60{ }^{\circ} \mathrm{C}$, human cells die almost immediately. Longer exposure at lower temperatures is needed to attain cell death (8). Larger ablation volume in a shorter time needs high temperatures. Though, temperatures larger than $100{ }^{\circ} \mathrm{C}$ yield tissue burning and evaporation which leads to lower current concentration and isolation, lessening both heat production and conduction 
which leads to cold parts and insufficient ablations.

Importantly, the area between the electrode return pads and the body is an additional site of production of heat. Skin burns may be the result of the lengthy application of RF energy near the border of the electrode return pads. Better dispersion of heat occurs with Pads which have larger surface areas.

\section{PATIENTS AND METHODS:}

The study was an interventional clinical trial approved by the scientific ethics committee. The study was conducted between September 2017 and February 2019. Forty women with symptomatic uterine myomas were enrolled and underwent USgRFA at Obs./Gyn. the department, Sohag University Hospital. The study was done in the operative theater of the department of Obstetrics and Gynaecology, Sohag university hospital in collaboration with the Radiology department.

The selection criteria for this study were as follows: (1)Premenopausal women; (2)Age $\geq 20$ years; (3)Fibroids producing irregular uterine bleeding, pelvic pain or discomfort, urinary or intestinal symptoms, or dyspareunia; (4)Fibroids which needs surgical intervention; (5)Size of Uterus equal or less than 16 weeks; (6)The myomas are submucosal or intramural or subserosal; (7) The myomas are $\geq 3 \mathrm{~cm}$ in diameter; (8)The patient is capable of giving written consent.

The exclusion criteria included infertility planned management, intracavitary pedunculated (FIGO Type 0) myoma, myomas FIGO Type 1 (submucosal with $\geq 50 \%$ intracavitary), scheduled associated surgery with treatment of uterine myomas, use of any metal device (eg, copper IUD), implantable apparatus inside the pelvis, recent Pregnancy, pelvic infection in the last 3 months, history of pelvic malignancy and/or pelvic radiation, doubt for dense pelvic adhesions, myomas managed by myomectomy, radio-frequency ablation, uterine artery embolism, cryomyolysis or MRg-FUS in the last 3 months, the existence of more than two fibroids, one fibroid greater than $7 \mathrm{~cm}$, abnormal results of a correlogram.

We use the Model 1500X Electrosurgical Radiofrequency Generator (Rita Medical Systems, USA). This provides monopolar radiofrequency and delivers 150 watts of RF power in most modes with up to 200 watts RF power in the infusion mode.

The preoperative evaluation: A thorough clinical evaluation (history taking and examination) was done to all patients, routine investigations, surgical fitness and precise abdominal and vaginal ultrasonographic evaluation of the number, size, and site of leiomyomas. Leiomyoma volume was estimated. Follow up was done by ultrasonography three and six months after treatment.

$\mathrm{We}$ use the following formula to calculate the leiomyoma volume: volume $=\pi / 6 \times \mathrm{D} 1 \times \mathrm{D} 2 \times \mathrm{D} 3$, where $\mathrm{D} 1$ is the longitudinal dimension, D2 is the anterior-posterior dimension, and D3 is the transverse one.

We used the RITA radiofrequency thermoablation needle(length $15 \mathrm{~cm}$, diameter $2.2 \mathrm{~mm}$ ), with 9 hooks at the extremity $3-5 \mathrm{~cm}$ (which mimics the configuration of a Christmas tree), which were opened during the procedure. In myomas $>5 \mathrm{~cm}$, two-needle placements were done to achieve a volume of necrosis equal to the volume of myomas. The needle probe was attached to a 
generator (Rita Medical Systems, USA) that works at extreme power of $150 \mathrm{~W}$ and at temperatures extending from 15 to $125^{\circ} \mathrm{C}$; the device exhibits the characteristics of tissue impedance plus the ablation power and time. The chosen temperature to attain in the tissue was $105 \mathrm{C}$ and the device automatically maintains that temperature $(150 \mathrm{~W})$ by adjusting the power.

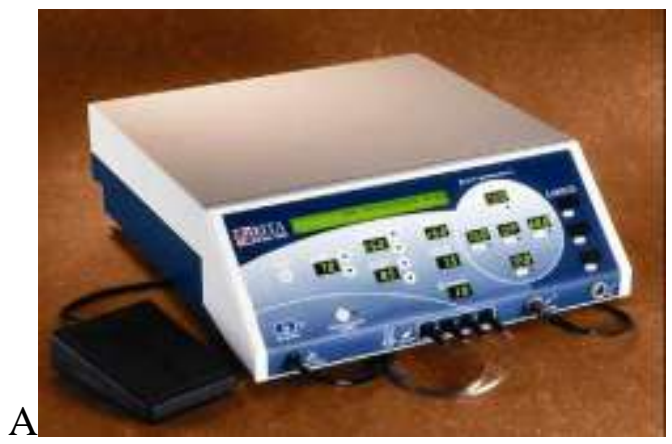

B

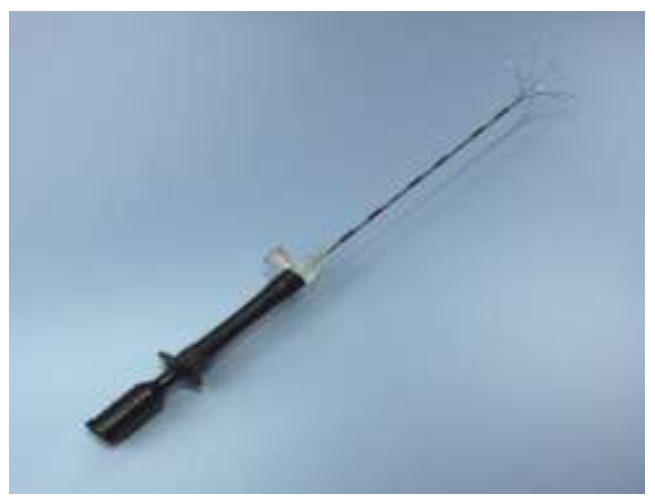

Fig.(1) A- Rita Electrosurgical Radiofrequency Generator Model 1500X B-The RITA radiofrequency thermoablation needle

Percutaneous RFA was performed under spinal anesthesia. One gram of 3rd generation cephalosporins was taken as a prophylactic antibiotic.

A real-time Ultrasound guide was used during the procedure(Albanian, Ecube5). In all patients, We assessed the existence of minor and major complications during and after the procedure. Minor complications are brief and self-limiting symptoms needing no treatment or normal treatment without any clinical complications. Major complications are those needing additional interventions and/or hospitalization.

In all patients, the technical achievement was obtained (100\%).

Primary Result measures: Changes in symptoms related to myoma, tumor size (volume) post-procedure and echogenicity of the tumor at baseline and three and six months after the procedure.

\section{Statistical analysis :}

The SPSS computer program version 20.0 was used to analyze the data. Mean \pm standard deviation, median and interquartile range were used to express the quantitative data. Number and percentage were used to express qualitative data. The Shapiro-Wilk test was used for quantitative data to test for normality. As data weren't normally distributed the nonparametric Friedman test and Wilcoxon Signed Ranks test were used. For normally distributed data a paired-samples t-test was used. Marginal Homogeneity test, Cochran's $\mathrm{Q}$ test, Friedman test, and McNemar test were used for comparing repeated measures of other qualitative variables. A 5\% level was taken as a level of significance in all statistical tests used in the study.

\section{Results:}

Between September 2017 and February 2019, 40 patients with symptomatic uterine leiomyomas, including abnormal uterine bleeding(38 patients) and pelvic pain(2 patients), were assessed at Sohag University Hospital. The number of patients was 40 (mean age, $38.75 \pm 7.25$ years; median age, 39 years) with 40 
leiomyomas fulfilled all inclusion criteria and underwent USgRFA and were observed to evaluate the effects. thirty-eight patients were subjected to ultrasound-guided radiofrequency ablation through the abdominal route, two patients were subjected to radiofrequency ablation for posterior uterine wall myoma in a retroverted uterus through the vaginal route and through the vaginal ultrasound guidance.

\begin{tabular}{|c|c|}
\hline Characteristics & Summary statistics \\
\hline Age (years) & $38.75 \pm 7.25$ \\
Mean \pm S.D. & $39(33.5-45)$ \\
Median (IQ range) & $9(22.5 \%)$ \\
\hline Parity & $14(35 \%)$ \\
Grand multipara & $4(10 \%)$ \\
Multipara & $2(5 \%)$ \\
Unipara & $5(12.5 \%)$ \\
Nullipara & $6(15 \%)$ \\
Nulligravida & \\
Unmarried & \\
\hline
\end{tabular}

Table (1): distribution of the studied patients according to age and parity (No.=40).

$40 \%$ of myomas were located in the anterior uterine wall, $32.5 \%$ are fundal in location, $27.5 \%$ are in the posterior uterine wall.

$87.5 \%$ of myomas were intramural, 10 $\%$ were submucous, $2.5 \%$ were subserous.

The procedure time ranged from 30 to 60 min. $($ mean $=45)$ dependent on the size of the myoma and the number of deployments of the needle. No complications had occurred intra. or postoperative during or following the procedure. All patients were discharged from the hospital after 3 hours of observation. None of the patients reported severe vaginal bleeding (defined as five or more soaked sanitary pads per day), fever, or dyspnea. Dull pain in the lower abdomen in 10 patients
(25\%) was relieved within 24 hours after the procedure with NSAIDs.

The follow-up time was 6 months after initial management. The mean volume of the leiomyoma and the decrease of the volume during the follow-up period are presented in table 2 and figure 2.

\begin{tabular}{|c|c|c|c|}
\hline $\begin{array}{l}\text { Volume } \\
\text { (cm3) }\end{array}$ & Pre-RFA & $\begin{array}{l}\text { Three months } \\
\text { post-RFA }\end{array}$ & $\begin{array}{l}\text { Six months } \\
\text { post-RFA }\end{array}$ \\
\hline $\begin{array}{c}\text { Mean } \pm \text { S.D. } \\
\text { Median } \\
\text { (IQ range) }\end{array}$ & $\begin{array}{ll}47.93 & \pm \\
40.55 & \\
& \\
33.49 & \\
(20.76 & - \\
61.82) & \end{array}$ & $\begin{array}{l}23.52 \pm \\
21.35 \\
14.6 \quad(9.16 \\
-32.21)\end{array}$ & $\begin{array}{l}14.21 \pm \\
15.5 \\
9.24(4.73 \\
-16.08)\end{array}$ \\
\hline
\end{tabular}

Table(2) comparison between the repeated tumor volume $\left(\mathrm{cm}^{3}\right)$ measures $($ No. $=40)$.

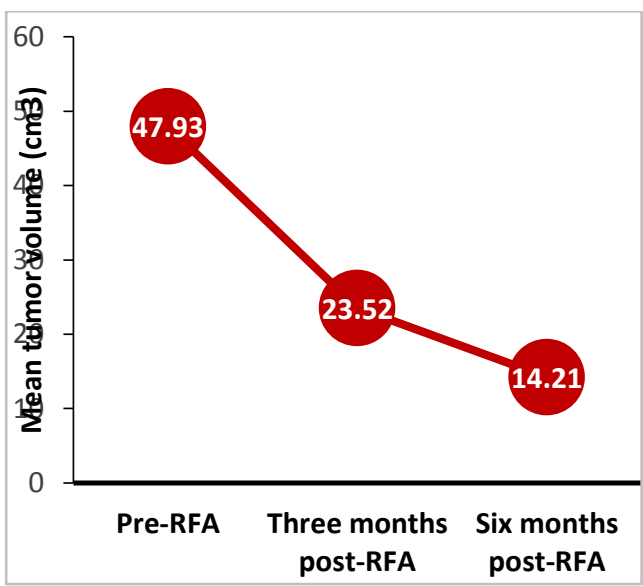

Figure (2): comparison between the repeated tumor volume $\left(\mathrm{cm}^{3}\right)$ measures.

All patients who accomplished the six months follow-up period were completely symptom-free.

\section{Discussion:}

In our study, we assessed the safety and efficiency of USgRF ablation in the management of symptomatic uterine leiomyomas, and the results showed that this procedure can effectively destroy leiomyomas, safe, feasible and without complications during or after the procedure in nearly all patients. The 
procedure is appropriate for day surgery settings because of the decreased level of described pain and the absence of adverse events. Uterine fibroid symptoms were significantly improved.

The first clinical experiment was conducted by Bergamini et al. 2005(9), radiofrequency ablation management via laparoscopy was done for eighteen patients with symptomatizing uterine myomas. The study highlighted the ablative effectiveness (for 9 women the size reduced by $85 \%$, the symptom severity score reduced, and the quality of life was better) and there are no immediate or late problems. The following trials by Ghezzi et al, 2007 and Milic et al, 2006 revealed equal results as much as the decrease in volume and improvements in symptoms. In the study by Ghezzi et al, the mean follow-up was 24 months, with a $70 \%$ decrease in volume of treated myomas at six months and constant results at the three-year follow-up. Kim et al, 2007, 35 women were treated with percutaneous radiofrequency ablation after performing uterine artery embolization to increase its clinical effectiveness. The mean decrease in volume was $56 \%$, and at the similar time symptoms had been better six months after treatment. The procedure was not effective in one patient; hysterectomy was done to two patients after RF management because of persistent pain at five and twelve months. In any study, no major complications had occurred. Recaldini C. et al, 2007(10), published a feasibility study, in which percutaneous radiofrequency ablation was done for 6 premenopausal women with symptomatic leiomyomas under abdominal ultrasonographic guidance. Symptoms relief and reduction in the volume and diameter of the leiomyomas were measured every three months. they decided that Percutaneous USgRFA alone is a practical and effective method in the treatment of medium-sized uterine leiomyomas. Brucker et al, 2015(11) published the result of a randomized, prospective clinical trial evaluating radiofrequency ablation of myomas with the Acessa System and laparoscopic myomectomy. Twenty-five patients were treated in each group. loss of blood during the technique and stay in hospital were both statistically significantly lower in the RFA group. Ninety-eight percent of myomas shown intraoperatively by laparoscopic ultrasound were treated in the radiofrequency ablation group versus $80 \%$ in the myomectomy group. The time of operation was also lesser in the RFA group.

On 8 Jan. 2019, Victoria E. et al(12) published an observational study of 205 patients with metrorrhagia secondary to type II/III submucosal or intramural cavity-distorting myomas undergoing outpatient transvaginal ultrasoundguided radiofrequency myolysis (TRFAM) under sedation between September 2015 and February 2017.

Complications during and after the procedure, correction of metrorrhagia, patient satisfaction, mean volume of myoma, and hemoglobin level at one, three, six, and twelve months after the process are the main result measures. At 12 months the mean volume reduction was $60 \%$ when compared with preoperative volume. All women had normal menstruation at a mean followup of 3 months .the study concluded that TRFAM is an efficient and safe technique in selected patients for the 
treatment of metrorrhagia secondary to myomas.

The results issued in the literature confirm our study, displaying that this technique might be feasible and efficient in decreasing the volume and ameliorating symptoms. we obtained a shrinkage rate of about 40 and $60 \%$ at the 3 and 6-month follow-up respectively.

Some limitations existed in our study, including the fact that we did not perform randomization, a control group lacking, a short follow-up period, and a small number of patients. These limitations may definitely weaken the effectiveness of the study outcome, making prospective randomized controlled clinical trials indispensable to verify the effect of USgRFA in the treatment of uterine leiomyomas. Because these patients did not have a reproduction requirement, we did not study the effect of RFA on pregnancy.

\section{Conclusion:}

Radiofrequency ablation of leiomyomas has confirmed to be a valuable alternative to major surgery, versatile, safe, and efficient in decreasing or eradicating symptoms associated with uterine leiomyomas and a low chance of recurrence after treatment. It is effective in the management of leiomyomas of different sizes and numbers in one setting.

Additional studies and longer follow-ups with large data to confirm the exact recurrence rate, long term complications, and effect on pregnancy is needed.

\section{References:}

1. Doina SJ, Minnie M, Sahadat N, William HC. Myometrial cells undergo fibrotic transformation under the influence of transforming growth factor $\beta-3$. Fertil Steril. 2010;93(5):1500-8.

2. Bulun SE. Uterine fibroids. $\mathrm{N}$ Engl $\mathrm{J}$ Med. 2013;369:1344-55.

3. Ciavattini A, Di Giuseppe J, Stortoni P, Montauk N, Giannubilo SR, Litta P et al. Uterine Fibroids: Pathogenesis and Interactions with Endometrium and Endomyometrial Junction. Obstetrics and Gynecology International Volume 2013, ArticleID 173184, 11 pages.

4. Sparic R, Mirkovic L, Malvasi A, Tinelli A. Epidemiology of uterine myomas: a review. Int J Fertil Steril. 2016;9(4):42435.

5. Olejek A, Olszak-Wąsik K, CzerwinskaBednarska A. Long-term intermittent pharmacological therapy of uterine fibroids - a possibility to avoid hysterectomy and its negative consequences. Menopause Rev. 2016;15(1):48-51.

6. Khan AT, Shehmar M. Gupta JK Uterine fibroids: current perspectives. Int J Women's Health. 2014;6:95-114.

7. Hammerich D. Tissue ablation. In: Webster JG, editor. Wiley encyclopedia of medical devices and instrumentation. New Jersey: John Wiley \& Sons; 2006. pp. 362-379.

8. Goldberg SN, Solbiati L, Hahn PF, et al. Large-volume tissue ablation with radiofrequency by using a clustered, internally-cooled electrode technique: laboratory and clinical experience in liver metastases. Radiology. 1998;209:371-9.

9. Bergamini V1, Ghezzi F, Cromi A, Bellini G, Zanconato G, Scarperi S, Franchi M. Laparoscopic radiofrequency thermal ablation: a new approach to symptomatic uterine myomas. Am J Obstet Gynecol. 2005 Mar;192(3):76873.

10. Recaldini C., Gianpaolo Carrafiello, et al. American Journal of Roentgenology. 2007;189:1303-1306. 10.2214/AJR.07.2184.

11. Brucker S, Hahn M, Kraemer D, Wallwiener M, Taran FA, Wallwiener 
SOHAG MEDICAL JOURNAL

Vol. 23 No. 3 July 2019

$\mathrm{CW}$, et al. Radiofrequency Volumetric Thermal Ablation of Fibroids and Laparoscopic Myomectomy: Long-Term Follow-up From a Randomized Trial. Geburtshilfe-Frauenheilkd.

2015;75(5):442-9.
Impact of Radiofrequency ablation in the management Elsemary M.A

12. Rey VE, Labrador R, Falcon M, GarciaBenitez JL. Transvaginal Radiofrequency Ablation of Myomas: Technique, Outcomes, and Complications. Journal of laparoendoscopic \& advanced surgical techniques Part A. 2019;29(1):24-8. 